\title{
Action = réaction
}

La «Caisse unique», comme j'ai pu le constater ces dernières semaines à l'occasion de plusieurs réunions et entretiens personnels, jouit d'un capital étonnant de sympathie dans le corps médical. Etonnant dès lors que, parmi les consœurs et confrères concernés (plusieurs à des postes-clé), nombre d'entre eux s'en tiennent généralement à des positions fermement bourgeoises quand il s'agit de voter ou d'élire. Mais un deuxième regard sur ce "changement de camp» est beaucoup moins surprenant. Ces opinions s'insèrent dans la mosaïque d'une image plus large qui reflète une situation actuelle en santé publique beaucoup plus dure qu'avant.

C'est une lapalissade de constater que la pression économique sur le corps médical a été en constante augmentation ces dernières années. Les médecins se voient de plus en plus confrontés à des devoirs économiques et de politique de la santé, qui touchent à des degrés divers leurs compétences premières, celles de traiter l'homme malade selon les règles de l'art médical. Cette tendance apparaît aussi dans le BMS: un indicateur est le nombre toujours croissant d'articles qui ont pour thèmes la gestion de la santé publique et l'optimalisation de l'économie d'entreprise. Au sein de la rédaction, nous pensons que refléter plus ou moins précisément ce développement est justifié dans la mesure où le BMS tient à entretenir et encourager un débat aussi proche de la réalité que possible entre les représentants de la santé publique. La question ne se pose pas: il est évident que les médecins font partie de ce monde-là et doivent se plier à ces réalités. Il n'y a pas de raison de penser qu'ils n'y sont pas prêts en leur plus grande majorité.

Le ton de plus en plus décidé du camp de l'économie de la santé a aussi un effet (annexe) que l'on peut qualifier de tout à fait positif. Cette tendance sort le corps médical du bois pour l'amener à opposer à la perspective économique une perspective médicale, dans laquelle les priorités ne sont par nature pas les mêmes. Il s'agit en fait de savoir quelle médecine nous voulons, car dans un cadre devenu incontestablement plus étroit, des possibilités existent encore de se diriger plutôt dans l'une direction que dans l'autre.

Ceux d'entre nous qui, ces dernières années, ont vécu ce développement «au front» comprennent le sentiment de nombre de médecins qui voient la bureaucratisation grandissante de la médecine entraîner de nombreux effets contreproductifs. Motivés avant tout par le principe d'efficacité, d'adéquation et d'économicité (EAE), les efforts de contrôle généralement sincères des instances responsables conduisent à des lenteurs administratives et des pressions de se justifier, très frustrantes pour nombre de médecins et de patients, les mesures médicales bien pensées et judicieuses ne pouvant pas toujours se fonder sur des arguments incontestables. Nombre de consœurs et confrères doutent que le système (de caisses-maladie) tel qu'il est établi serve la santé des patients à long terme.

La boucle étant bouclée, revenons à la caisse unique. Il n'est pas injustifié de penser que nombre de médecins se plaisent à prôner cette solution non parce qu'ils en sont fondamentalement convaincus, mais davantage par dépit face au système actuel. C'est précisément en vue de mesures médicales efficaces à long terme que le corps médical met certains espoirs dans une caisse unique, qui suivrait «ses» clients à vie. Nous ne nous demanderons pas ici si de tels espoirs sont fondés ou non. Mais la question mérite d'être posée.

Dans ce contexte, il sera sans doute passionnant de suivre les débats de politique de santé sur le sujet de la caisse unique lors de la séance de la Chambre médicale des 14 et 15 décembre prochains. Le résultat - la position officielle de la FMH - fera sûrement office de signal et sera comme de juste publié dans le BMS.

Bruno Kesseli 